\title{
Kinematics Comparative Study of Two Overconstrained Parallel Manipulators
}

\author{
Qiang Yan, ${ }^{1}$ Bin Li, ${ }^{1,2}$ Yangmin $\mathrm{Li}^{1,2}$ and Xinhua Zhao' \\ ${ }^{1}$ Tianjin Key Laboratory for Advanced Mechatronic System Design and Intelligent Control, Tianjin University of Technology, \\ Tianjin, China \\ ${ }^{2}$ Faculty of Science and Technology, University of Macau, Taipa, Macau \\ Correspondence should be addressed to Bin Li; cnrobot@163.com
}

Received 27 June 2016; Revised 12 August 2016; Accepted 21 September 2016

Academic Editor: Alberto Borboni

Copyright (C) 2016 Qiang Yan et al. This is an open access article distributed under the Creative Commons Attribution License, which permits unrestricted use, distribution, and reproduction in any medium, provided the original work is properly cited.

\begin{abstract}
A comparison study of kinematics characteristics of two overconstrained 2-RPU\&SPR parallel manipulators (PMs) is introduced in this paper. The two 2-RPU\&SPR PMs have the same kinematics properties in terms of one translational degree of freedom (DOF) and two rotational DOFs kinematics outputs. But there are some differences between the two PMs as far as joints distribution is concerned, leading to the differences in respect of workspace and dexterity of the two PMs. Firstly, based on screw theory, the structural characteristics and DOFs of the two PMs are analyzed. Secondly, the inverse and forward displacements problems for the two PMs are formulated by analytic formulae. Some numerical examples are simulated by software. Thirdly, based on algorithm for the direct displacement solution, the workspace characteristics of the two PMs are analyzed and compared. Then, the Jacobian matrices of the mechanisms are formulated. Based on the Jacobian matrices, the dexterities of the two PMs are established and compared. Finally, according to the comparisons of the properties between the two PMs, some useful conclusions are provided.
\end{abstract}

\section{Introduction}

In recent years, lower-mobility PMs have played a more important role in both services and industrial applications. Compared to 6-DOF parallel manipulators, the lowermobility structures have simpler mechanical design, lower manufacturing cost, and larger workspace. In terms of characteristics of DOFs, the lower-mobility PMs can be classified into three categories, namely, pure translational PMs, pure rotational PMs, and mixed PMs. Compared to the top two types PMs, the mixed PMs have both translational DOFs and rotational DOFs, and this type of PMs displays good potential for future applications in the field of motion simulator or a mixed orientating/positioning tool. Many scholars have done some research on this type of PMs and developed their own design.

In 1983, Hunt [1] developed a 3-RPS PM, which was a classical mixed PM, and the related research work about the 3-RPS PM could be found in many literatures. The kinematic analyses concerning positon analysis, synthesis, dynamic modeling, workspace analysis, instantaneous motions analysis, and so forth can be found in the literature [2-8]. According to changeable layout angle of actuators $\mathrm{Li}$ and $\mathrm{Xu}$ [9] developed a parallel manipulator, which is called 3-PRS $\mathrm{PM}$, and the kinematic performances of the mechanism are analyzed. Liu et al. [10-12] presented a type of new 3-DOF $\mathrm{PM}$ which has two translational freedoms and one rotational freedom with three nonidentical chains. The inverse and forward kinematics problems and singularity problems for this type PMs are presented. Recently, Li et al. [13] proposed a novel overconstrained 2-RPU\&SPR PM, and the kinematic performance of the PM is analyzed. Yang [14] developed a family of 1T2R PMs with three limbs in his monograph. Qi et al. [15] discussed the kinematic optimum design of the 3PUS/PU with 1T2R DOFs PM. Apart from the mixed 3-DOF PMs mentioned above, some other similar 3-DOF structures can be seen in the literature [16-19].

The comparison study of two overconstrained 2RPU\&SPR parallel manipulators, which have owned one translational DOF and two rotational DOFs, is analyzed in 


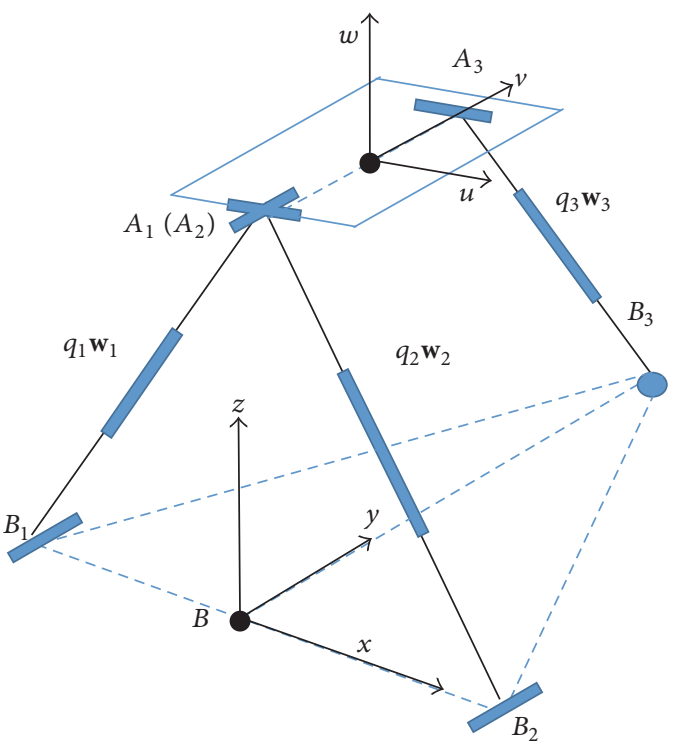

FIGURE 1: Type one 2-Rㅁ&SPRR PM.



FIgURE 2: Type two 2-RㅁU\&SㅁR PM.

this paper. The content is disposed in the following structure. Firstly, based on screw theory, the structural characteristics and DOFs of the two PMs are described in Sections 2 and 3, respectively. Some numerical examples are depicted in Section 4. In Section 5, the workspace concerning the two PMs is acquired by using the algorithm for the direct displacement solution, and some conclusions are concluded. The general Jacobian matrix for the two PMs is obtained, and the dexterity comparison is analyzed in Section 6. According to the analysis of the platform motion performances and based on the two structures, some conclusions and future studies are drawn in Section 7.

\section{Structure Characteristics of the Two Overconstrained 2-RP U\&SPR Manipulators}

Type one 2-RPU\&SPR PM involves a static plate and a mobile platform which is connected by two same RPU limbs and one SPR limb as shown in Figure 1. Here, R, U, and $S$ are the abbreviation of the revolute, universal, and spherical joints. The driving prismatic joints are $\mathrm{P}$ which are underlined. Placing the reference frame $B-x y z$ on the base, similarly, the mobile frame $A-u v w$ is set on the mobile plate. Points $B$ and $A$ are middle points of lines $B_{1} B_{2}$ and $A_{1} A_{3}$, respectively. The axes of $x$ and $u$ are separately parallel to $B_{1} B_{2}$ and $A_{1} A_{2}$, the axes of $z$ and $w$ are perpendicular to the base platform and the moving platform, respectively. $B_{i}(i=1,2)$ and $A_{3}$ are the intersection of the axes of the revolute joints and actuated prismatic joints, $B_{3}$ is the middle point of the spherical joint, and the centroid of the universal joints is $A_{1}$ and $A_{2}$ separately. It should be extraordinarily obviously noted that, for type one 2-RPU\&SPR PM, the universal joints of the RPU limbs which are connected on the moving platform are shared.

Here, type two 2-RPU\&SPR PM has two different universal joints for RPU limbs relative to type one 2-RPU\&SPR PM. The value of the distance concerning the two universal joints is $A_{1} A_{2}$ in Figure 2 .

The direction matrix of the $A-u v w$ relative to the $B-x y z$ frame can be found by using three Euler angles $\psi, \phi$, and $\theta$, and the three parameters are satisfying $x-z-y$ conventions conditions:

$$
\begin{aligned}
\mathbf{R} & =\left[\begin{array}{ccc}
c \theta c \phi & s \psi s \theta-c \psi c \theta s \phi & c \psi s \theta+s \psi c \theta s \phi \\
s \phi & c \psi c \phi & -s \psi c \phi \\
-s \theta c \phi & s \psi c \theta+c \psi s \theta s \phi & c \psi c \theta-s \psi s \theta s \phi
\end{array}\right] \\
& =\left[\begin{array}{lll}
u_{x} & v_{x} & w_{x} \\
u_{y} & v_{y} & w_{y} \\
u_{z} & v_{z} & w_{z}
\end{array}\right]=\left[\begin{array}{lll}
\mathbf{u} & \mathbf{v} & \mathbf{w}
\end{array}\right] .
\end{aligned}
$$

Here, the unit vectors of the frame $A-u v w$ relative to the frame $B-x y z$ are $\mathbf{u}, \mathbf{v}$, and $\mathbf{w}$; " $\mathrm{s}$ " and " $\mathrm{c}$ " are the abbreviations of sin and cos.

\section{Mobility Analysis of the Two Mechanisms via Screw Theory}

Reciprocal screw theory is used for analyzing constraint forces, which is exerted on the mobile plate, in this paper. As for a parallel manipulator, the reciprocal screws associated with a serial limb stand for the constraints of wrench. These wrench constraints exerted on the mobile platform are realized through serial limbs. Using the properties of the reciprocal screw theory, the outcomes of mobility analysis concerning the two type 2-RPU\&SPR PMs will not depend on the change of coordinate system. 


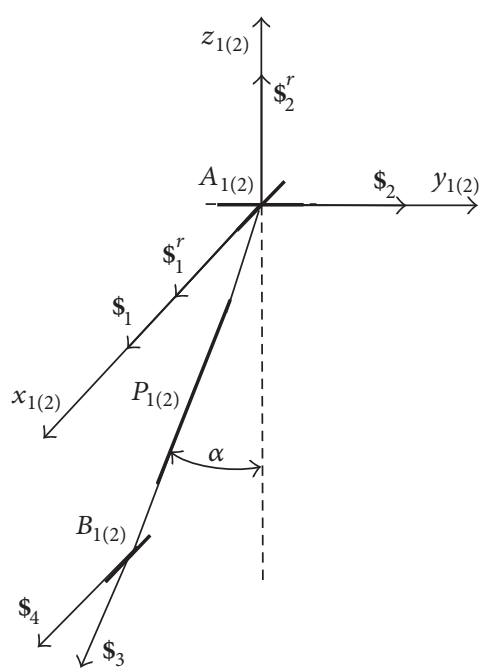

(a) RPU limb

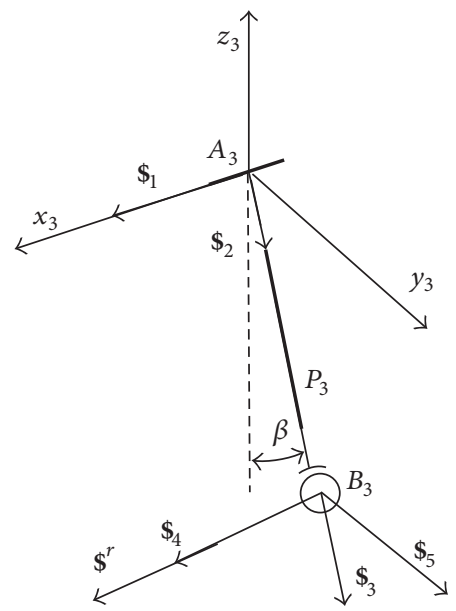

(b) SㅁR limb



Taking into account the versatility, each limb is set for a local coordinate system $A_{i}-x_{i} y_{i} z_{i}, i=1,2,3$ in Figure 3. The limb twist system of the RPU limb in Figure 3(a) is given by

$$
\begin{aligned}
& \$_{1}=\left(\begin{array}{llllll}
1 & 0 & 0 & 0 & 0 & 0
\end{array}\right), \\
& \$_{2}=\left(\begin{array}{llllll}
0 & 1 & 0 & 0 & 0 & 0
\end{array}\right), \\
& \$_{3}=\left(\begin{array}{llllll}
0 & 0 & 0 & 0 & -\sin \alpha-\cos \alpha
\end{array}\right), \\
& \$_{4}=\left(\begin{array}{llllll}
1 & 0 & 0 & 0 & -q_{1(2)} \cos \alpha & q_{1(2)} \sin \alpha
\end{array}\right),
\end{aligned}
$$

where $q_{i}=\left\|A_{i} B_{i}\right\|, i=1,2,3$.

Considering the reciprocity between twist and wrench, the constraint system for the R $\underline{P} \mathrm{U}$ limb can be described as

$$
\begin{aligned}
& \boldsymbol{\$}_{1}^{r}=\left(\begin{array}{llllll}
1 & 0 & 0 & 0 & 0 & 0
\end{array}\right), \\
& \$_{2}^{r}=\left(\begin{array}{llllll}
0 & 0 & 0 & 0 & 0 & 1
\end{array}\right) .
\end{aligned}
$$

Here, $\$_{1}^{r}$ denotes a constraint force, it is passing through the centroid of universal joint and paralleling to the axis of joint screw $\$_{1}$, and $\$_{2}^{r}$ is the abbreviation of constraint couple. The direction of the constraint couple is norm to the axes of joint screws $\$_{1}$ and $\$_{2}$ which are passing through the centroid of the joints.

In Figure 3(b), the limb twist system concerning the SPR limb can be described as follows:

$$
\begin{aligned}
& \mathbf{\$}_{1}=\left(\begin{array}{llllll}
1 & 0 & 0 & 0 & 0 & 0
\end{array}\right), \\
& \mathbf{\$}_{2}=\left(\begin{array}{llllll}
0 & 0 & 0 & 0 & \sin \beta & -\cos \beta
\end{array}\right), \\
& \$_{3}=\left(\begin{array}{llllll}
0 & \sin \beta & -\cos \beta & 0 & 0 & 0
\end{array}\right), \\
& \$_{4}=\left(\begin{array}{llllll}
1 & 0 & 0 & 0 & -q_{3} \cos \beta & -q_{3} \sin \beta
\end{array}\right), \\
& \mathbf{\$}_{5}=\left(\begin{array}{llllll}
0 & -\cos \beta & -\sin \beta & -q_{3} & 0 & 0
\end{array}\right) .
\end{aligned}
$$

The constraint screw concerning the limb of SPR can be described:

$$
\$^{r}=\left(\begin{array}{llllll}
1 & 0 & 0 & 0 & -q_{3} \cos \beta & -q_{3} \sin \beta
\end{array}\right) .
$$

The system in (5) demonstrates a constraint force passing through the spherical joint center and it is parallel to the axis of $\$_{1}$ in each configuration.

Using (3) and (5), we can know that two RPU limbs constrain a translational DOF of the mobile plate. The direction is the same as the constraint force. Meanwhile, it restricts a rotational DOF and the direction is the same as the constraint couple. Similarly, the SPR restricts a translational DOF of the mobile platform along the direction of the constraint force. So, the two types 2-RPU\&SPR PM have the same DOFs, which include one translational DOF and two rotational DOFs.

\section{Inverse and Forward Position Analyses}

4.1. Inverse Position Analysis. Inverse position analysis of the two PMs involves the determination of the limb lengths given the pose of the mobile platform.

The vector $\mathbf{r}=\left(\begin{array}{lll}x & y & z\end{array}\right)^{\mathrm{T}}$ which represents point $A$ in the $B-x y z$ can be described by using the loop closure equation for both type 2-RPU\&SPR PMs.

$$
\begin{aligned}
& \mathbf{r}=\mathbf{b}_{i}+q_{i} \mathbf{w}_{i}+\overrightarrow{A_{i} A_{0}}-\mathbf{a}_{i} \quad i=1,2, \\
& \mathbf{r}=\mathbf{b}_{i}+q_{i} \mathbf{w}_{i}-\mathbf{a}_{i} \quad i=3 .
\end{aligned}
$$

Here, $\mathbf{a}_{i}=\mathbf{R a}_{i 0}$, the length and unit vector of limb $i$ are $q_{i}$, and $\mathbf{w}_{i}$ respectively, and the position vectors of $A_{i}$ are $\mathbf{a}_{10}=$ $a_{1}\left(\begin{array}{lll}-k & 0 & 0\end{array}\right)^{\mathrm{T}}, \mathbf{a}_{20}=a_{2}\left(\begin{array}{lll}k & 0 & 0\end{array}\right)^{\mathrm{T}}$, and $\mathbf{a}_{30}=a_{3}\left(\begin{array}{lll}0 & -1 & 0\end{array}\right)^{\mathrm{T}}$, respectively, which is measuring in $A-u v w$. The position vectors of $B_{i}$ are $\mathbf{b}_{1}=b_{1}\left(\begin{array}{lll}-1 & 0 & 0\end{array}\right)^{\mathrm{T}}, \mathbf{b}_{2}=b_{2}\left(\begin{array}{lll}1 & 0 & 0\end{array}\right)^{\mathrm{T}}$, and $\mathbf{b}_{3}=b_{3}\left(\begin{array}{lll}0 & 1 & 0\end{array}\right)^{\mathrm{T}}$ which are measured in $B$-xyz. $a_{i}$ and $b_{i}$ are 
the values of distance concerning $\left\|A A_{i}\right\|$ and $\left\|B B_{i}\right\|, a_{1}=a_{2}=$ $a_{3}=a, b_{1}=b_{2}=b$. For type one 2-RPU\&SPR PM, $k=0$. For type two 2-RPU\&SPR PM, $k=1$.

The constraint concerning the RPU limb is imposed by the revolute joint; it restricts the vectors of $\mathbf{w}_{i}$ and $\mathbf{b}_{i}$; meanwhile, it is normal to the unit vector $\mathbf{c}$ which represents the axis of the revolute joint. So, on both sides of (6), making the dot product with $\mathrm{c}$ yields

$$
\left(\mathbf{r}+\mathbf{a}_{i}\right)^{\mathrm{T}} \mathbf{c}=0, \quad i=1,2 .
$$

Here, $\mathbf{c}=\left(\begin{array}{lll}0 & 1 & 0\end{array}\right)^{\mathrm{T}}$.

In the same way, the constraint of the SPR limb which is exerted on the revolute joint restricts the vectors of $\mathbf{w}_{3}$ and $\mathbf{a}_{3}$; meanwhile, it is perpendicular to the vector $\mathbf{c}_{3}$ which is the axis of revolute joint. So, on both sides of (6), making the dot product with $\mathbf{c}_{3}$ yields

$$
\left(\mathbf{r}-\mathbf{b}_{i}\right)^{\mathrm{T}} \mathbf{c}_{3}=0, \quad i=3 .
$$

Here, $\mathbf{c}_{3}=\mathbf{R}_{30}, \mathbf{c}_{30}=\left(\begin{array}{lll}1 & 0 & 0\end{array}\right)^{\mathrm{T}}$.

Based on the geometric constraint of the two PMs, as shown in Figure 2, the axis of the joint $R 2$ can just only move in the plane $B x z$, we can get another equation, and it can be listed as follows:

$$
u_{y}=0
$$

Through (7), (8), and (9), the equation which concerns the two PMs can be listed as follows:

$$
\begin{aligned}
& \phi=0, \\
& y=a \cos (\psi), \\
& x=z \tan (\theta) .
\end{aligned}
$$

Here, $\phi, y$, and $x$ stand for the parasitic motions which is relative to the mobile plate. The parasitic motions concerning the two PMs can contribute to perplex kinematics, require real-time compensation, increase difficulty in calibration, and even lead to damage. Given a set of unconstrained variables $\left(\begin{array}{lll}\psi & \theta & z\end{array}\right)$, the parasitic motion of the two PMs can be computed by using (10), (11), and (12). Then, the value of length concerning the limb can be listed as follows:

$$
q_{i}=\left|\mathbf{r}-\mathbf{b}_{i}+\mathbf{a}_{i}\right|, \quad i=1,2,3 .
$$

4.2. Forward Position Analysis of the Two Mechanisms. The forward position analysis concerning the two PMs involves the determination of the pose of the mobile plate. Firstly, on both sides of (13), making Euclidean norm and taking some necessary addition or subtraction, the simplified equations can be presented as follows:

$$
\begin{aligned}
q_{1}^{2}= & {[z \tan (\theta)-t \cos (\theta)-a \sin (\psi) \sin (\theta)+b]^{2} } \\
& +[z+t \sin (\theta)-a \sin (\psi) \cos (\theta)]^{2}
\end{aligned}
$$

$$
\begin{aligned}
q_{2}^{2}= & {[z \tan (\theta)+t \cos (\theta)-a \sin (\psi) \sin (\theta)-b]^{2} } \\
& +[z-t \sin (\theta)-a \sin (\psi) \cos (\theta)]^{2}, \\
q_{3}^{2}= & {[z \tan (\theta)+a \sin (\psi) \sin (\theta)]^{2} } \\
& +\left[2 a \cos (\psi)-b_{3}\right]^{2} \\
& +[z+a \sin (\psi) \cos (\theta)]^{2} .
\end{aligned}
$$

(1) When $q_{1} \neq q_{2}$, through subtraction and addition with respect to (14) and (15), simplifying the equations, we can acquire

$$
\begin{aligned}
z= & f_{1} c \tan (\theta)+a \sin (\psi) \cos (\theta) \\
q_{1}^{2}+q_{2}^{2}= & \frac{2 z^{2}}{\cos (\theta)^{2}}-\frac{4 a z \sin (\psi)}{\cos (\theta)}+2 a^{2} \sin (\psi)^{2}+2 b^{2} \\
& +2 t^{2}-4 b t \cos (\theta) .
\end{aligned}
$$

Here, $f_{1}=\left(q_{1}^{2}-q_{2}^{2}\right) / 4 b$.

Then, substituting (17) into (18) and (16) gives

$$
\begin{aligned}
& 4 b t \cos ^{3}(\theta)-m \cos ^{2}(\theta)+4 b t \cos (\theta)=2 f_{1}^{2}-m \\
& q_{3}^{2} \\
& \quad=\frac{f_{1}^{2}}{\sin (\theta)^{2}}+4 a f_{1} \frac{\sin (\psi)}{\sin (\theta)}-4 a b_{3} \cos (\psi)+4 a^{2} \\
& \quad+b_{3}^{2},
\end{aligned}
$$

where $m=q_{1}^{2}+q_{2}^{2}-2 t^{2}-2 b^{2}$.

(2) When $q_{1}=q_{2}$, doing some subtractions with respect to (14) and (15), the equations can be simplified as follows:

$$
\theta=0
$$

Meanwhile, (14) and (16) can be rearranged as follows:

$$
\begin{aligned}
z & =\sqrt{q_{1}^{2}-(b-t)^{2}}+a \sin (\psi), \\
q_{3}^{2} & =\left[2 a \cos (\psi)-b_{3}\right]^{2}+[z+a \sin \psi]^{2} .
\end{aligned}
$$

Substituting (22) into (23) yields

$$
\begin{aligned}
q_{3}^{2}= & 4 a^{2}+q_{1}^{2}-(b-t)^{2}+b_{3}^{2}-4 a b_{3} \cos (\psi) \\
& +4 a \sin (\psi) \sqrt{q_{1}^{2}-(b-t)^{2}} .
\end{aligned}
$$

Accordingly, given a set of values $\left(\begin{array}{lll}q_{1} & q_{2} & q_{3}\end{array}\right)$, when $q_{1} \neq$ $q_{2}$, through using (19) and (22), the values of $\theta$ and $\psi$ can be computed. So, the direction matrix of $\mathbf{R}$ can be determined; through (12), (11), and (17), the values of $x, y$, and $z$ can be gotten. When $q_{1}=q_{2}$, through (21) and (24), the values of $\theta$ and $\psi$ can be computed. Similarly, the direction matrix of $\mathbf{R}$ can be acquired; in the same way, through (12), (11), and (22), the values of $x, y$, and $z$ can be obtained. 
TABLE 1: Inverse position analysis for type one mechanism.

\begin{tabular}{|c|c|c|c|c|c|c|c|c|}
\hline & \multicolumn{3}{|c|}{ Inputs } & \multicolumn{5}{|c|}{ Outputs } \\
\hline & $\psi$ (deg.) & $\theta$ (deg.) & $z(\mathrm{~mm})$ & $q_{1}(\mathrm{~mm})$ & $q_{2}(\mathrm{~mm})$ & $q_{3}(\mathrm{~mm})$ & $x(\mathrm{~mm})$ & $y(\mathrm{~mm})$ \\
\hline Case (a) & 25 & 35 & 700 & 1014.5651 & 685.7525 & 951.7624 & 490.1453 & 90.6308 \\
\hline Case (b) & -25 & 35 & 700 & 1096.7629 & 765.2621 & 872.5787 & 490.1453 & 90.6308 \\
\hline Case (c) & 25 & -35 & 700 & 685.7525 & 1014.5651 & 951.7624 & -490.1453 & 90.6308 \\
\hline Case $(\mathrm{d})$ & -25 & -35 & 700 & 765.2621 & 1096.7629 & 872.5787 & -490.1453 & 90.6308 \\
\hline
\end{tabular}

TABLE 2: Forward position analysis for type one mechanism.

\begin{tabular}{lcccccccc}
\hline & \multicolumn{3}{c}{ Inputs } & \multicolumn{3}{c}{ Outputs } \\
& $q_{1}(\mathrm{~mm})$ & $q_{2}(\mathrm{~mm})$ & $q_{3}(\mathrm{~mm})$ & $\psi($ deg. $)$ & $\theta($ deg. $)$ & $x(\mathrm{~mm})$ & $y(\mathrm{~mm})$ & $z(\mathrm{~mm})$ \\
\hline Case (a) & 653 & 816 & 941 & 61.0059 & -17.1845 & -225.3812 & 48.4719 & 728.7883 \\
Case (b) & 816 & 653 & 941 & 61.0059 & 17.1845 & 225.3812 & 48.4719 & 728.7883 \\
\hline
\end{tabular}

So the analyses of inverse and the forward position concerning the two PMs can be computed directly from Sections 4.1 and 4.2; meanwhile, the parasitic motions concerning the mobile plate can be described in a simplified way, which is enormously significant for mechanisms.

4.3. Numerical Examples. The concerned parameters of the two PMs are defined as follows: $a=100 \mathrm{~mm}, b=300 \mathrm{~mm}$, and $b_{3}=500 \mathrm{~mm}$. Based on the inverse position analysis relative to type one, given four different groups of inputs $\left(\begin{array}{lll}\psi & \theta & z\end{array}\right)$, through computing, the corresponding parameters are listed in Table 1; meanwhile, the corresponding configurations relative to the computed solutions are described in Figure 4. In the same way, as for the forward position analysis, given two different groups of inputs $\left(\begin{array}{lll}q_{1} & q_{2} & q_{3}\end{array}\right)$, output parameters are computed and listed in Table 2; the corresponding configurations relative to the solutions are described in Figure 5. Similarly, given four different groups of inputs $\left(\begin{array}{lll}\psi & \theta & z\end{array}\right)$ to type two PMs, the corresponding results are computed and described in Table 3, and the corresponding poses relative to results are described in Figure 6. Given two different groups of inputs $\left(\begin{array}{lll}q_{1} & q_{2} & q_{3}\end{array}\right)$ to forward position analysis, the output parameters concerning type two PMs are listed in Table 4, and the corresponding configurations concerning the computed solutions are depicted in Figure 7.

\section{The Workspace Analysis and Comparison of the Two Mechanisms}

Given a group of limb lengths $\left(\begin{array}{lll}q_{1} & q_{2} & q_{3}\end{array}\right)$, through corresponding equations in Section 4 , the other parameters involving parasitic motions are computed directly. So when the restrictions to the limb lengths are set up, the parasitic motion workspace and the reachable workspace of the two mechanisms can be obtained directly. Accordingly, some comparisons concerning the two PMs are obtained.

5.1. Case Studies and Comparison of the Two Mechanisms. The concerning parameters of two PMs are defined as follows: $a=100 \mathrm{~mm}, b=300 \mathrm{~mm}, b_{3}=500 \mathrm{~mm}, t=100 \mathrm{~mm}$, $600 \mathrm{~mm} \leqslant q_{1} \leqslant 900 \mathrm{~mm}, 600 \mathrm{~mm} \leqslant q_{2} \leqslant 900 \mathrm{~mm}$, and $600 \mathrm{~mm} \leqslant q_{3} \leqslant 900 \mathrm{~mm}$. The workspace concerning two PMs is analyzed by software. In Figures 8 and 9, the workspace of parasitic motion concerning the two PMs is described. The reachable workspace along the directions of $x$-, $y$-, $z$-axes are depicted in Figures 10,11 , and 12 . The positional workspace of the mobile plate is described in Figure 13.

From Figures 8 to 13, we can see that the reachable spaces of the two types of the 2-R $\underline{U} U \& S \underline{P R}$ PMs are similar in shape, but there exists some difference in size. For type one 2-RPU\&SPR PM, the reachable workspace of the moving platform in the direction of $x, y$, and $z$ is bigger than that of type one PM, and the volume of the reachable space of type one PM is bigger than that of type two.

\section{The Dexterity Analysis and Comparison of the Two Mechanisms}

The velocity of each limb can be gotten by differentiating (6), so the equations can be described as follows:

$$
\dot{\mathbf{r}}=\dot{q}_{i} \mathbf{w}_{i}+\boldsymbol{\omega}_{i} \times q_{i} \mathbf{w}_{i}-\boldsymbol{\omega} \times \mathbf{a}_{i} \quad i=1,2,3 .
$$

Here, the velocity of $i$ th linear actuator is $\dot{q}_{i}$, the angular velocity of link $A_{i} B_{i}$ is $\omega_{i}$, " $\times$ " represent the cross product between vectors, and $\dot{\mathbf{r}}=\left(\begin{array}{lll}\dot{x} & \dot{y} & \dot{z}\end{array}\right)^{\mathrm{T}}$ and $\boldsymbol{\omega}=\left(\begin{array}{lll}\omega_{x} & \omega_{y} & \omega_{z}\end{array}\right)^{\mathrm{T}}$ stand for the linear and angular velocity of the mobile plate, respectively.

Through dot multiplying on both sides of (25) with $\mathbf{w}_{i}$, the passive variables $\boldsymbol{\omega}_{i}$ are eliminated; this yields

$$
\dot{q}_{i}=\mathbf{w}_{i} \dot{\mathbf{r}}+\left(\mathbf{a}_{i} \times \mathbf{w}_{i}\right) \cdot \boldsymbol{\omega} \quad i=1,2,3 .
$$

If the two PMs are far from singularities, we can rewrite (26) in the matrix form:

$$
\dot{\mathbf{q}}_{a}=\mathbf{J}_{a} \dot{\mathbf{X}}
$$

Here, $\dot{\mathbf{q}}_{a}=\left[\begin{array}{c}\dot{q}_{1} \\ \dot{q}_{2} \\ \dot{q}_{3}\end{array}\right], \dot{\mathbf{X}}=\left[\begin{array}{c}\dot{r} \\ \boldsymbol{\omega}\end{array}\right], \mathbf{J}_{a}=\left[\begin{array}{c}\mathbf{w}_{1}^{\mathrm{T}}\left(\mathbf{a}_{1} \times \mathbf{w}_{1}\right)^{\mathrm{T}} \\ \mathbf{w}_{2}^{\mathrm{T}}\left(\mathbf{a}_{2} \times \mathbf{w}_{2}\right)^{\mathrm{T}} \\ \mathbf{w}_{3}^{\mathrm{T}}\left(\mathbf{a}_{3} \times \mathbf{w}_{3}\right)^{\mathrm{T}}\end{array}\right]$.

The values of inverse velocity concerning the PMs can be computed by (27). Because the two PMs described in the 
TABLE 3: Inverse position analysis for type one mechanism.

\begin{tabular}{lcccccccc}
\hline & \multicolumn{3}{c}{ Inputs } & & \multicolumn{3}{c}{ Outputs } \\
& $\psi($ deg. $)$ & $\theta($ deg. $)$ & $z(\mathrm{~mm})$ & $q_{1}(\mathrm{~mm})$ & $q_{2}(\mathrm{~mm})$ & $q_{3}(\mathrm{~mm})$ & $x(\mathrm{~mm})$ & $y(\mathrm{~mm})$ \\
\hline Case (a) & 25 & 35 & 700 & 995.0845 & 727.6026 & 957.0014 & 490.1453 \\
Case (b) & -25 & 35 & 700 & 1078.8 & 802.9790 & 878.2902 & 490.1453 \\
Case (c) & 25 & -35 & 700 & 656.5877 & 1043.3 & 957.0014 & -490.1453 & 90.6308 \\
Case (d) & -25 & -35 & 700 & 739.2408 & 1123.4 & 872.5787 & -490.1453 & 90.6308 \\
\hline
\end{tabular}

TABLE 4: Forward position analysis for type one mechanism.

\begin{tabular}{lcccccccc}
\hline & \multicolumn{3}{c}{ Inputs } & \multicolumn{4}{c}{ Outputs } \\
& $q_{1}(\mathrm{~mm})$ & $q_{2}(\mathrm{~mm})$ & $q_{3}(\mathrm{~mm})$ & $\psi($ deg. $)$ & $\theta($ deg. $)$ & $x(\mathrm{~mm})$ & $y(\mathrm{~mm})$ & $z(\mathrm{~mm})$ \\
\hline Case (a) & 653 & 816 & 941 & 50.4875 & 16.3288 & -212.3484 & 97.5455 \\
Case (b) & 816 & 653 & 941 & 50.4875 & 16.3288 & 186.7299 & 97.5455 & 261.9190 \\
\hline
\end{tabular}

paper possess only 3-DOF, we can obviously pay attention to the fact that the linear and angular velocity components of the mobile plate are not all independent.

Through differentiating (10), (11), and (12) with respect to time, respectively, we can get the parasitic motions equations:

$$
\begin{aligned}
& \dot{\phi}=0, \\
& \dot{y}=-a \sin (\psi) \dot{\psi}, \\
& \dot{x}=\dot{z} \tan (\theta)+z \sec ^{2}(\theta) \dot{\theta} .
\end{aligned}
$$

Through differentiating the direction matrix $\mathbf{R}$ concerning time, the equations can be gotten:

$$
\dot{\mathbf{R}}=\mathbf{S}(\omega) \mathbf{R},
$$

where $\mathbf{S}(\omega)=\left[\begin{array}{ccc}0 & -\omega_{z} & \omega_{y} \\ \omega_{z} & 0 & -\omega_{x} \\ -\omega_{y} & \omega_{x} & 0\end{array}\right]$.

Then, through (32) and using $\dot{\psi}$ and $\dot{\theta}, \boldsymbol{\omega}=\left(\begin{array}{lll}\omega_{x} & \omega_{y} & \omega_{z}\end{array}\right)^{\mathrm{T}}$ are described:

$$
\boldsymbol{\omega}=\left[\begin{array}{cc}
\cos \theta & 0 \\
0 & 1 \\
-\sin \theta & 0
\end{array}\right]\left[\begin{array}{c}
\dot{\psi} \\
\dot{\theta}
\end{array}\right]
$$

Let $\dot{\mathbf{x}}=\left(\begin{array}{lll}\dot{\psi} & \dot{\theta} & \dot{z}\end{array}\right)^{\mathrm{T}}$; the implied relations are described by using (29), (30), (31), and (32):

$$
\dot{\mathbf{X}}=\mathbf{J}_{r} \dot{\mathbf{x}}
$$

Here $\mathbf{J}_{r}=\left[\begin{array}{ccc}0 & z \sec ^{2} \theta & \tan \theta \\ -\operatorname{asin} \psi & 0 & 0 \\ 0 & 0 & 1 \\ \cos \theta & 0 & 0 \\ 0 & 1 & 0 \\ -\sin \theta & 0 & 0\end{array}\right]$.

Through (27) and (33), the equations of relation can be obtained:

$$
\dot{\mathbf{q}}=\mathbf{J} \dot{\mathbf{x}}
$$

where $\mathbf{J}=\mathbf{J}_{a} \mathbf{J}_{r}$ is the Jacobian matrix of the mechanism, which includes the effect of the mechanical constrains on the mechanism. J is called the constrained Jacobian matrix of 2RPU\&SPR SPM.

From (34), the Jacobian matrix of type one 2-RPU\&SPR PM can be obtained as follows:

$$
\mathbf{J}_{T 1}=\left[\begin{array}{lll}
A_{11} & A_{12} & A_{13} \\
A_{21} & A_{23} & A_{24} \\
A_{31} & A_{32} & A_{33}
\end{array}\right],
$$

where

$$
\begin{aligned}
& A_{11}=\frac{a b \cos \psi \sin \theta-a^{2} \sin \psi \cos \psi}{J_{1}}, \\
& A_{12}=\frac{z \sec ^{2} \theta(a \sin \psi \sin \theta-b)+a b \sin \psi \cos \theta}{J_{1}}, \\
& A_{13}=\frac{(-b+a \sin \psi \sin \theta) \tan \theta+a \sin \varphi \cos \theta}{J_{1}}, \\
& A_{21}=\frac{-a b \sin \theta \cos \psi-a^{2} \sin \psi \cos \psi}{J_{2}}, \\
& A_{22}=\frac{z \sec { }^{2} \theta(b+a \sin \psi \sin \theta)-a b \sin \psi \cos \theta}{J_{2}}, \\
& A_{23}=\frac{\tan \theta(b+a \sin \psi \sin \theta)+a \sin \psi \cos \theta}{J_{2}}, \\
& A_{31}=\frac{-2 a b_{3} \sin \psi+a^{2} \sin \psi \cos \psi}{J_{3}}, \\
& A_{32}=\frac{-a z \sin \psi \sin \theta \sec { }^{2} \theta}{J_{3}}, \\
& J_{3}
\end{aligned}
$$




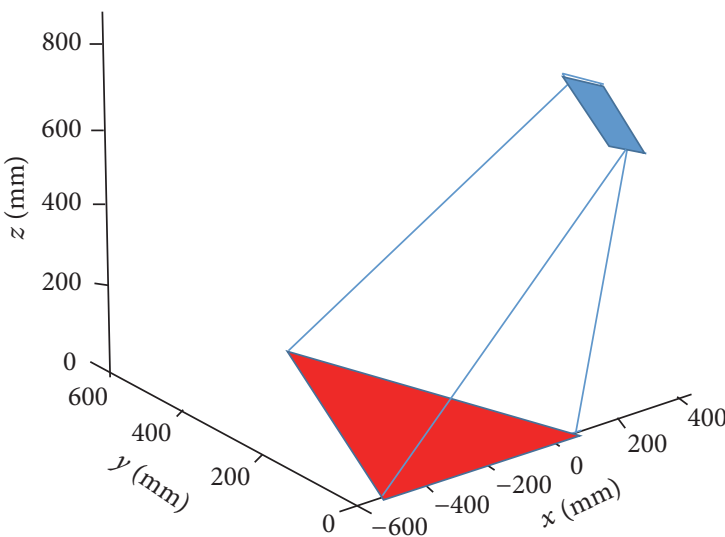

(a)



(c)

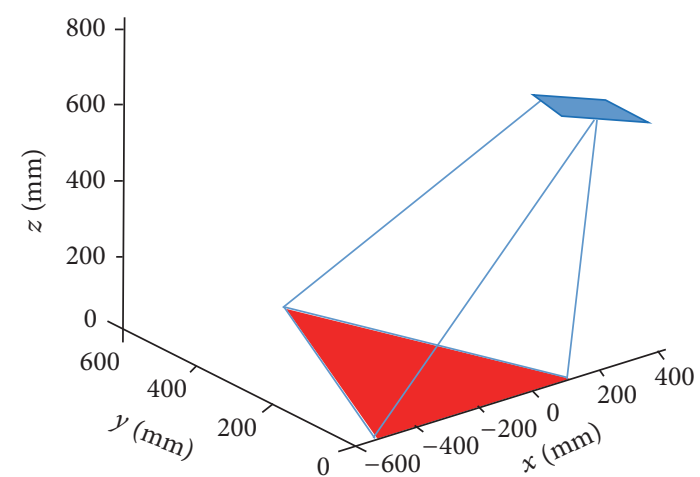

(b)

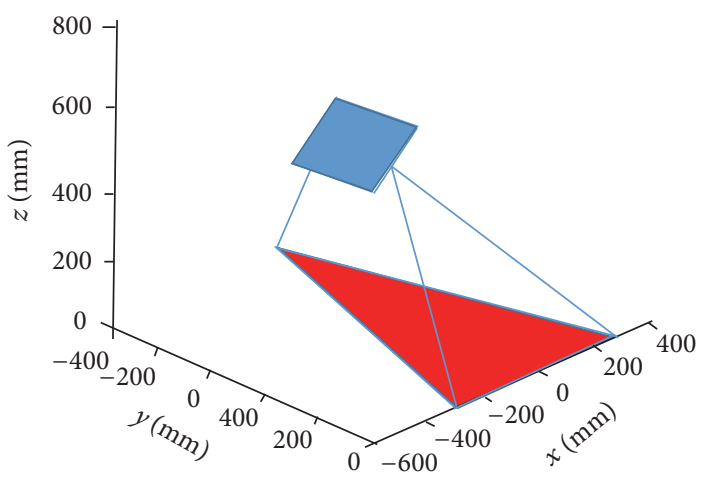

(d)

FIGURE 4: Different configuration solutions for inverse position analysis of type one 2-RPU\&SPR PM.

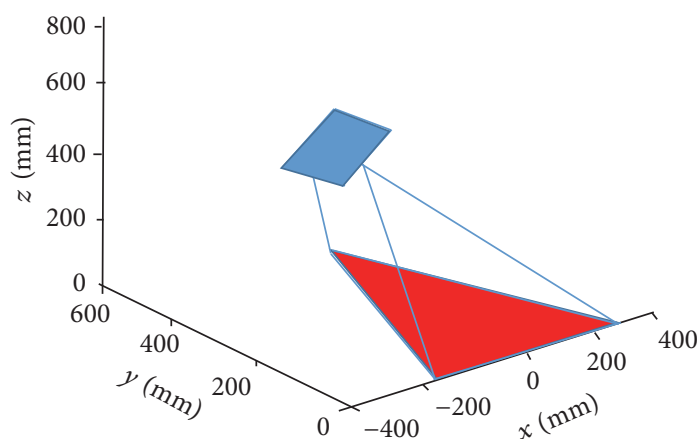

(a)

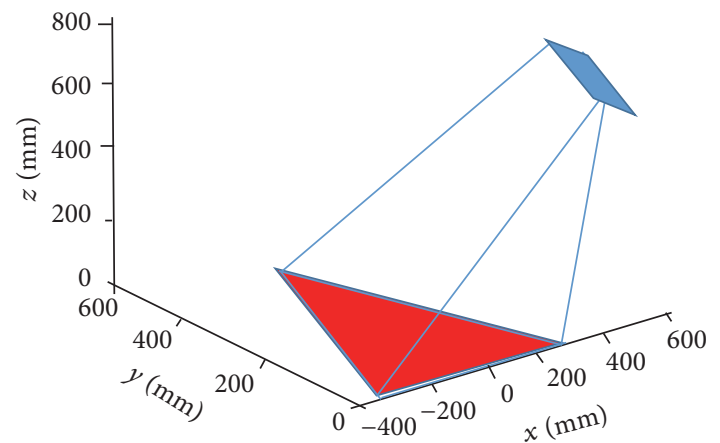

(b)

FIGURE 5: Different configuration solutions for inverse position analysis of type one 2-RPU\&SPR PM.

$$
\begin{aligned}
& J_{1}=\sqrt{a^{2}+b^{2}-2 a b \sin \psi \sin \theta}, \\
& J_{2}=\sqrt{a^{2}+b^{2}+2 a b \sin \psi \sin \theta}, \\
& J_{3}=\sqrt{a^{2}+b_{3}^{2}-2 a b_{3} \cos \psi} .
\end{aligned}
$$

The Jacobian matrix of type two 2-R $\underline{P U} \& \underline{S} \underline{P}$ PM can be calculated as follows:

$$
J_{T 2}=\left[\begin{array}{lll}
M_{11} & M_{12} & M_{13} \\
M_{21} & M_{22} & M_{23} \\
M_{31} & M_{32} & M_{33}
\end{array}\right] \text {, }
$$






(a)

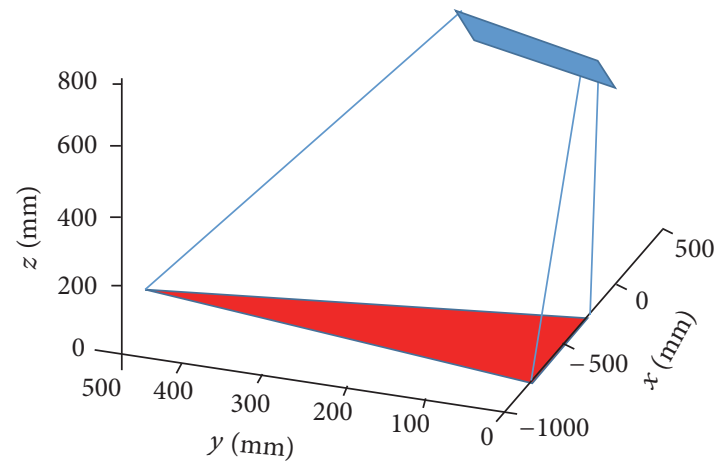

(c)

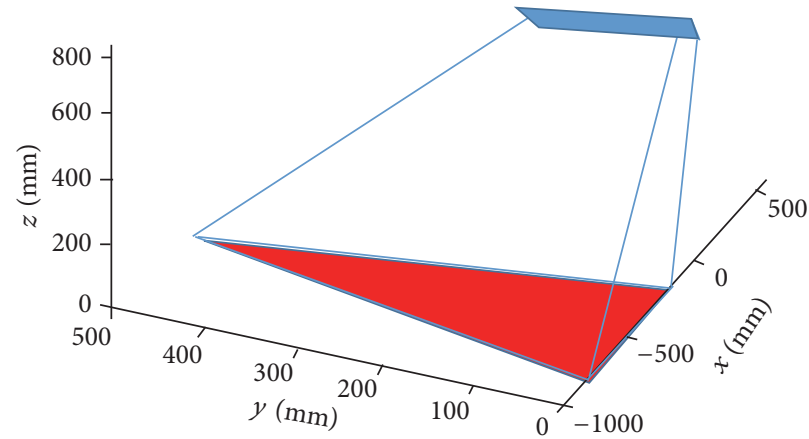

(b)

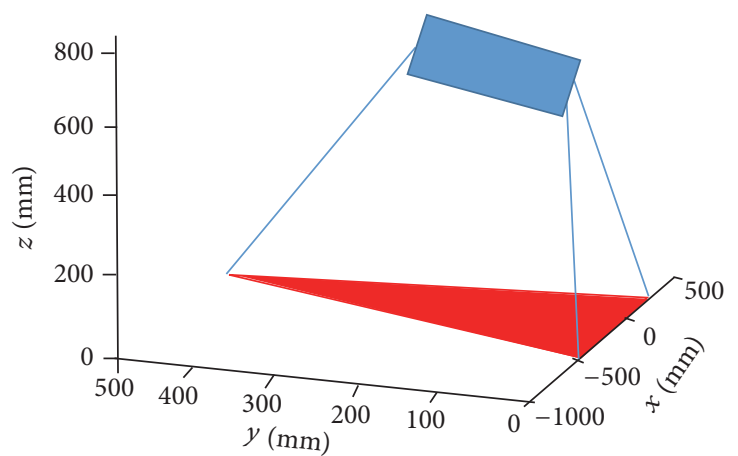

(d)

FIGURE 6: Different configuration solutions for inverse position analysis of type two 2-R $\underline{P} U$ \&S $\underline{P R}$ PM.

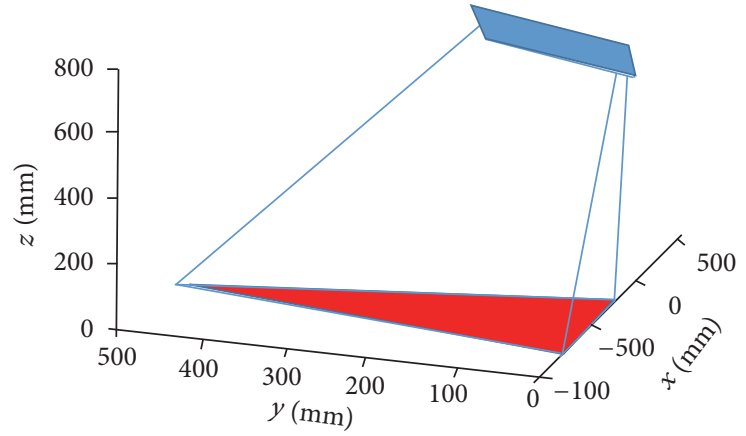

(a)

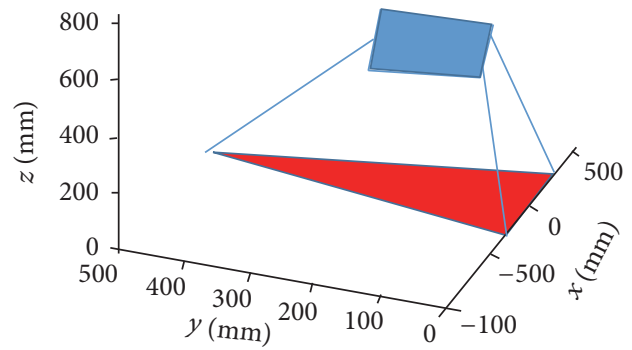

(b)

FIGURE 7: Different configuration solutions for forward position analysis of type two 2-RㅁU\&SPR PM.

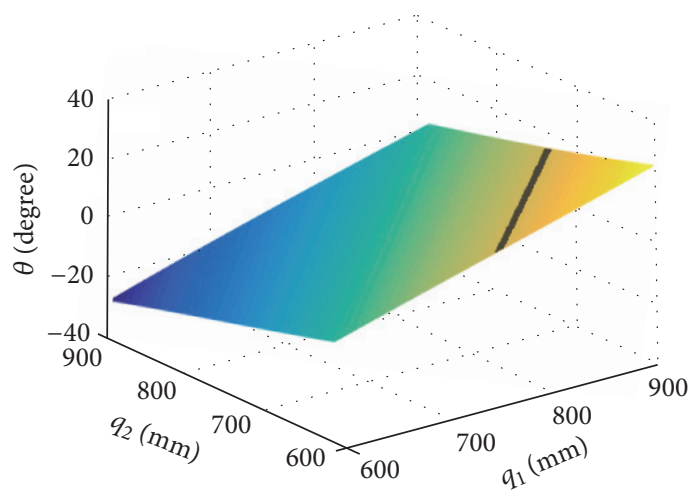

(a) Type one 2-RPU\&SPR PM

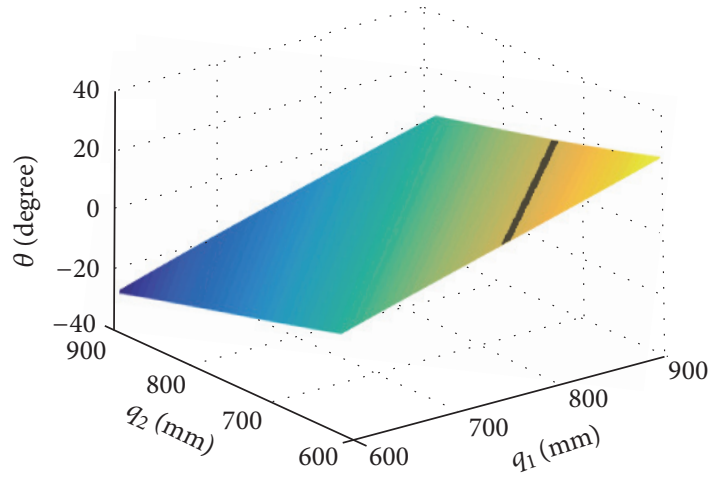

(b) Type two 2-RPU\&SPR PM

Figure 8: The distribution of $\theta$. 


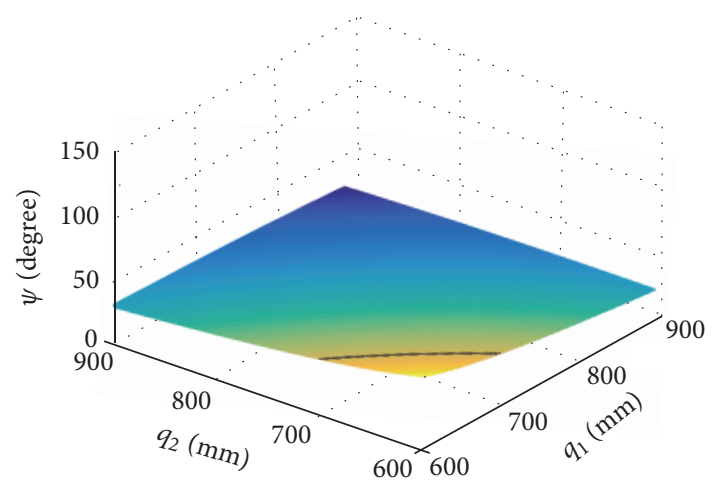

(a) Type one 2-RPU\&SPR PM

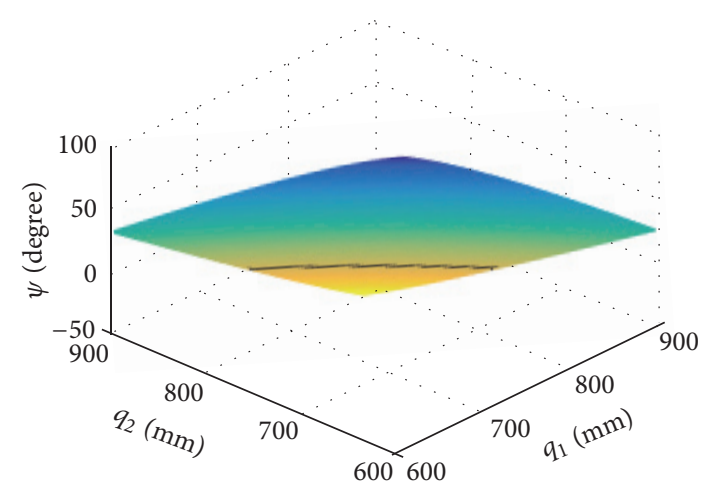

(b) Type two 2-Rㅁ&\&SR PM

Figure 9: The distribution of $\psi$.

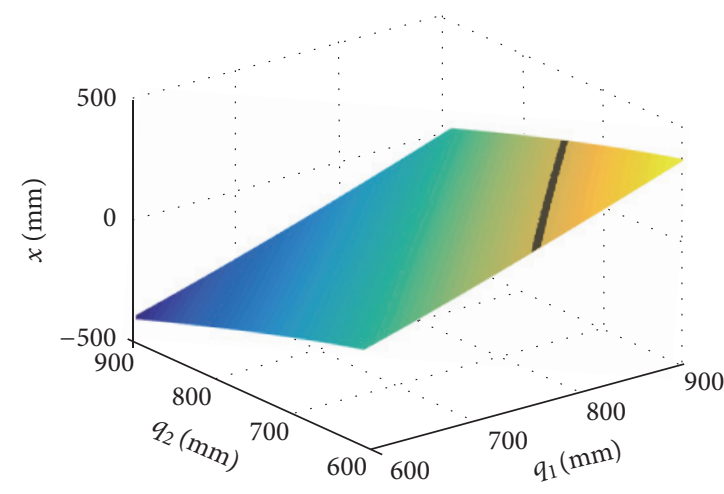

(a) Type one 2-RPU\&SPR PM



(b) Type two 2-RPU\&SPR PM

FIgURE 10: The reachable workspace in $x$-axis.

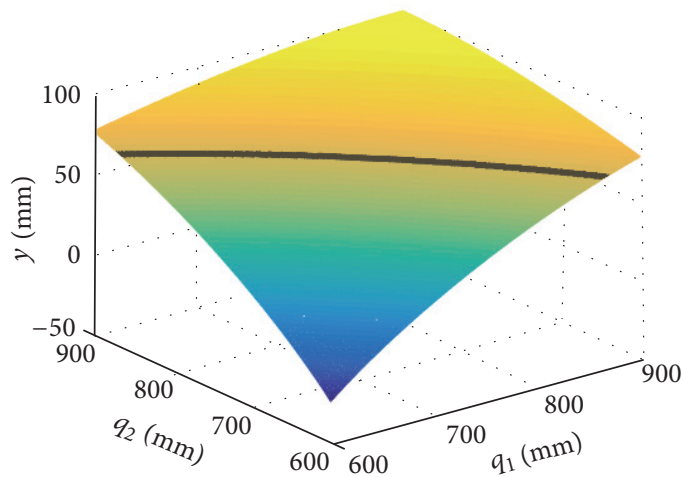

(a) Type one 2-Rㅁ&\&SR PM

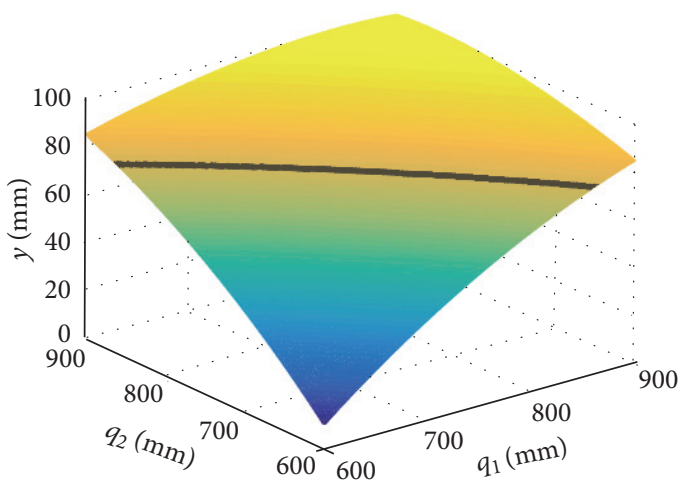

(b) Type two 2-RPUU\&SPR PM

FIGURE 11: The reachable workspace in $y$-axis.

where

$$
\begin{aligned}
& M_{11}=\frac{-a^{2} \sin \psi \cos \psi+a b \sin \theta \cos \psi}{D_{1}}, \\
& M_{12}=\frac{z(-b-a \cos \theta+a \sin \psi \sin \theta) \sec ^{2} \theta+a b \sin \psi \cos \theta+a b \sin \theta}{D_{1}},
\end{aligned}
$$




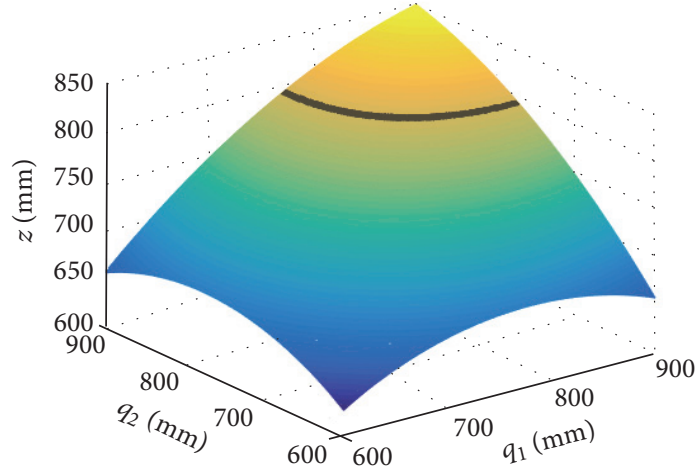

(a) Type one 2-RPU\&SPR PM

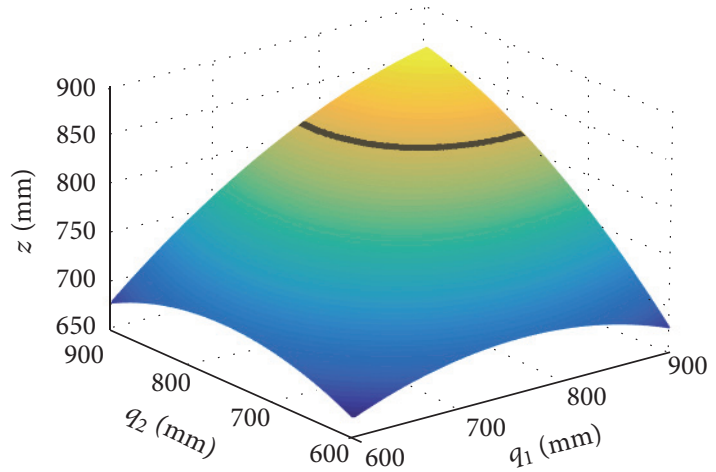

(b) Type two 2-RPU\&SPR PM

FIGURE 12: The reachable workspace in $z$-axis.

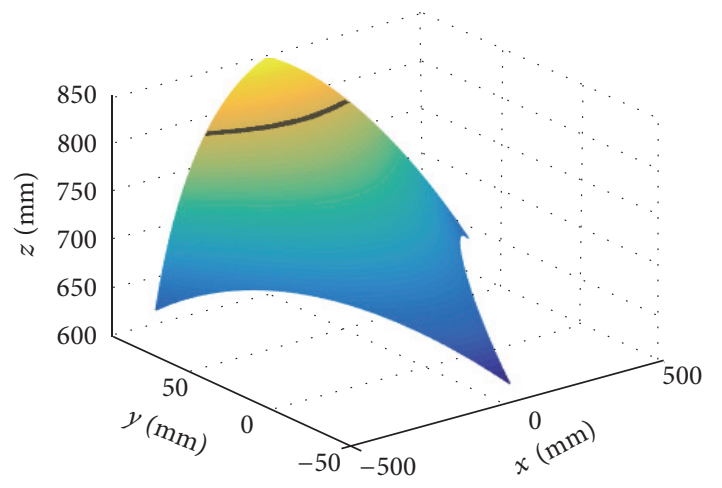

(a) Type one 2-RPU\&SPR PM

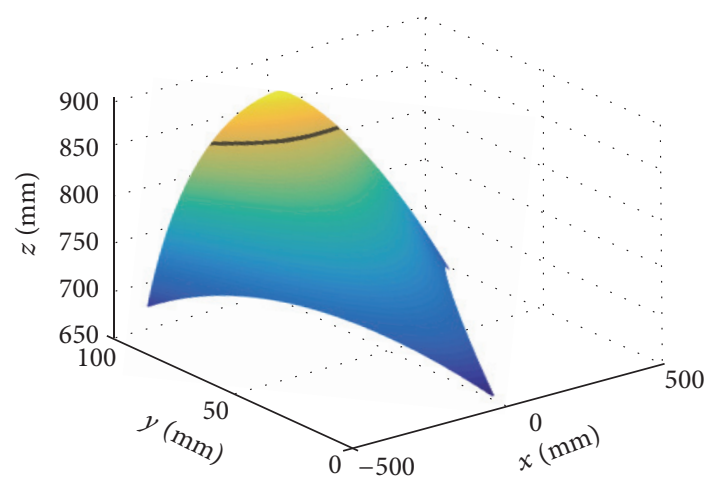

(b) Type two 2-RPU\&SR PM

FIGURE 13: The reachable workspace of the PMs.

$$
\begin{aligned}
& M_{13}=\frac{\tan \theta(-b-a \cos \theta+a \sin \psi \sin \theta)+a \sin \theta+a \cos \theta \sin \psi}{D_{1}}, \\
& M_{21}=\frac{-a^{2} \sin \psi \cos \psi-a b \sin \theta \cos \psi}{D_{2}}, \\
& M_{22}=\frac{z \sec ^{2} \theta(b+a \cos \theta+a \sin \psi \sin \theta)+a b \sin \theta-a b \cos \theta \sin \psi}{D_{2}}, \\
& M_{23}=\frac{\tan \theta(b+a \cos \theta+a \sin \psi \sin \theta)-a \sin \theta+a \sin \psi \cos \theta}{D_{2}}, \\
& M_{31}=\frac{a^{2} \sin \psi \cos \psi}{D_{3}}, \\
& M_{32}=\frac{-a z \sin \theta \sec \theta \sin \psi}{D_{3}}, \\
& M_{33}=\frac{-a \sin \theta \tan \theta \sin \psi-a \sin \psi \cos \theta}{D_{3}},
\end{aligned}
$$




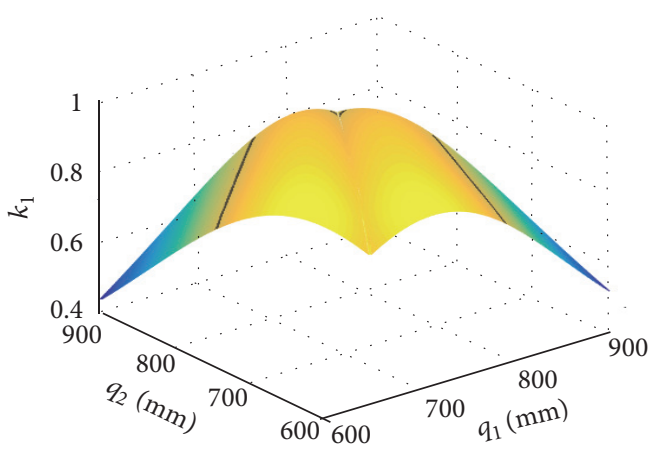

(a) Type one 2-RPU\&SPR PM

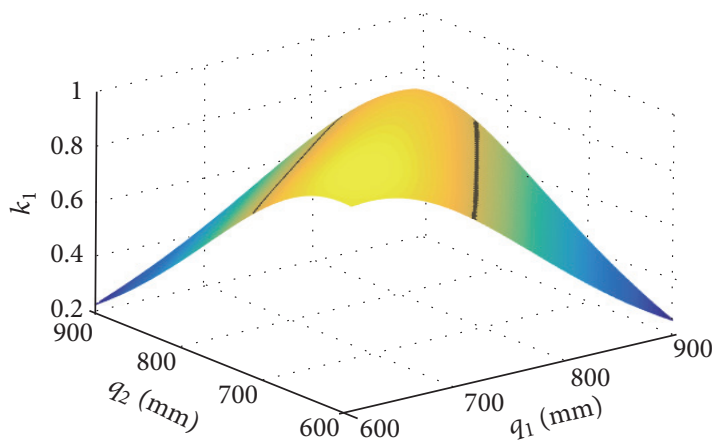

(b) Type two 2-RPU\&SPR PM

FIgURE 14: The dexterity of the PMs.

$$
\begin{aligned}
& D_{1}=\sqrt{2 a^{2}+b^{2}+2 a b \cos \theta-2 a b \sin \theta \sin \psi}, \\
& D_{2}=\sqrt{2 a^{2}+b^{2}+2 a b \cos \theta+2 a b \sin \theta \sin \psi}, \\
& D_{3}=\sqrt{a^{2}+b_{3}^{2}-2 a b_{3} \cos \psi} .
\end{aligned}
$$

From Figure 14, we can see that the distributions of dexterity of the two types PMs are similar in shape, but there exists some difference. The dexterity of type one 2-RPU\&SPR $\mathrm{PM}$ is better than that of type two PM.

\section{Conclusions}

This paper investigates the comparison study of two overconstrained 2-RPU\&SPR PMs. The two 2-RPU\&SPR PMs have identical kinematics limbs but different in joints distribution. The structure characteristics and DOFs of the two PMs are analyzed based on screw theory. Both of the inverse and forward displacements of the two PMs are derived by analytic formulae and some numerical examples are depicted. Unlike most parallel robots, these two overconstrained 2-RPU\&SPR PMs have explicit solutions for inverse and forward kinematics issues. The reachable workspaces of the two mechanisms are developed based on the forward position analysis and comparison analysis is carried out. The Jacobian matrix of the two PMs is derived, and dexterity comparison studies are provided. The stiffness and dynamics comparison of the two overconstrained 2-RPU\&SPR PMs will be studied in the future.

\section{Competing Interests}

The authors declare that they have no competing interests.

\section{Acknowledgments}

This work was supported by the National Natural Science Foundation of China (51575544, 51205289, and 51275353), the
Natural Science Foundation of Tianjin (14JCZDJC39100 and 16JCZDJC38000), and Science and Technology Major Project of Tianjin (15ZXZNGX00040 and 15ZXZNGX00270).

\section{References}

[1] K. H. Hunt, "Structural kinematics of in-parallel-actuated robot-arms," Journal of Mechanisms, Transmissions, and Automation in Design, vol. 105, no. 4, pp. 705-712, 1983.

[2] K. J. Waldron, M. Raghavan, and B. Roth, "Kinematics of a hybrid series-parallel manipulation system," Journal of Dynamic Systems, Measurement and Control, Transactions of the ASME, vol. 111, no. 2, pp. 211-221, 1989.

[3] S.-M. Song and M.-D. Zhang, "Study of reactional force compensation based on three-degree-of freedom parallel platforms," Journal of Robotic Systems, vol. 12, no. 12, pp. 783-794, 1995.

[4] Z. Huang, J. Wang, and Y. F. Fang, "Analysis of instantaneous motions of deficient-rank 3-RPS parallel manipulators," Mechanism and Machine Theory, vol. 37, no. 2, pp. 229-240, 2002.

[5] Y. Fang and Z. Huang, "Kinematics of a three-degree-offreedom in-parallel actuated manipulator mechanism," Mechanism and Machine Theory, vol. 32, no. 7, pp. 789-796, 1997.

[6] A. Sokolov and P. Xirouchakis, "Kinematics of a 3-DOF parallel manipulator with an R-P-S joint structure," Robotica, vol. 23, no. 2, pp. 207-217, 2005.

[7] J. Schadlbauer, D. R. Walter, and M. L. Husty, "The 3-RPS parallel manipulator from an algebraic viewpoint," Mechanism and Machine Theory, vol. 75, pp. 161-176, 2014.

[8] N. Farhat, V. Mata, Á. Page, and F. Valero, "Identification of dynamic parameters of a 3-DOF RPS parallel manipulator," Mechanism and Machine Theory, vol. 43, no. 1, pp. 1-17, 2008. 
[9] Y. Li and Q. Xu, "Kinematic analysis of a 3-PRS parallel manipulator," Robotics and Computer-Integrated Manufacturing, vol. 23, no. 4, pp. 395-408, 2007.

[10] X.-J. Liu, J. Wang, C. Wu, and J. Kim, "A new family of spatial 3-DOF parallel manipulators with two translational and one rotational DOFs," Robotica, vol. 27, no. 2, pp. 241-247, 2009.

[11] X.-J. Liu and J. Kim, "A new spatial three-DoF parallel manipulator with high rotational capability," IEEE/ASME Transactions on Mechatronics, vol. 10, no. 5, pp. 502-512, 2005.

[12] F. Xie, X.-J. Liu, and J. Wang, "A 3-DOF parallel manufacturing module and its kinematic optimization," Robotics and Computer-Integrated Manufacturing, vol. 28, no. 3, pp. 334-343, 2012.

[13] B. Li, Y. Li, X. Zhao, and Y. Yang, "Design and analysis of a spatial 2-RPU\&SPR parallel manipulator with 1T2R-type," in Proceedings of the 13th International Conference on Control Automation Robotics and Vision (ICARCV '14), pp. 1882-1887, Singapore, December 2014.

[14] T. L. Yang, Topology Structure Design of Robot Mechanisms, Machinery Industry Press, Beijing, China, 2003.

[15] M. Qi, H.-T. Liu, J.-P. Mei, X.-M. Zhao, and T. Huang, "Kinematics optimum design of a 3-DOF parallel mechanism with 3PUS/PU architecture," Journal of Tianjin University Science and Technology, vol. 40, no. 6, pp. 649-654, 2007.

[16] B. Li, Y. Li, X. Zhao, and W. Ge, "Comparative study of two 3CRU translational parallel manipulators," in Proceedings of the 2014 IEEE International Conference on Robotics and Biomimetics (IEEE ROBIO '14), pp. 332-337, IEEE, Bali, Indonesia, December 2014.

[17] B. Li, Y. M. Li, X. H. Zhao, and W. M. Ge, "Kinematic analysis of a novel 3-CRU translational parallel mechanism," Mechanical Sciences, vol. 6, no. 1, pp. 57-64, 2015.

[18] Y. M. Li and Q. S. Xu, "Kinematic analysis and design of a new 3DOF translational parallel manipulator," Journal of Mechanical Design, Transactions of the ASME, vol. 128, no. 4, pp. 729-737, 2006.

[19] Y. Li and Q. Xu, "Kinematics and dexterity analysis for a novel 3-DOF translational parallel manipulator," in Proceedings of the IEEE International Conference on Robotics and Automation, pp. 2944-2949, Barcelona, Spain, April 2005. 


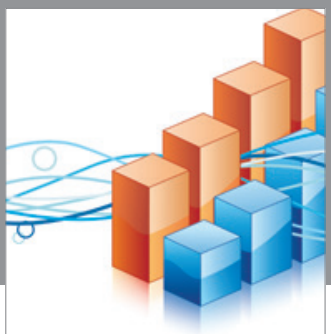

Advances in

Operations Research

vatem alat4

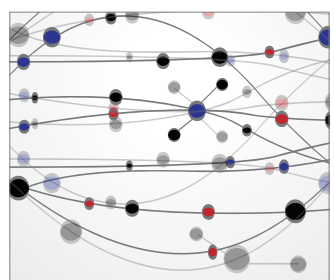

\section{The Scientific} World Journal
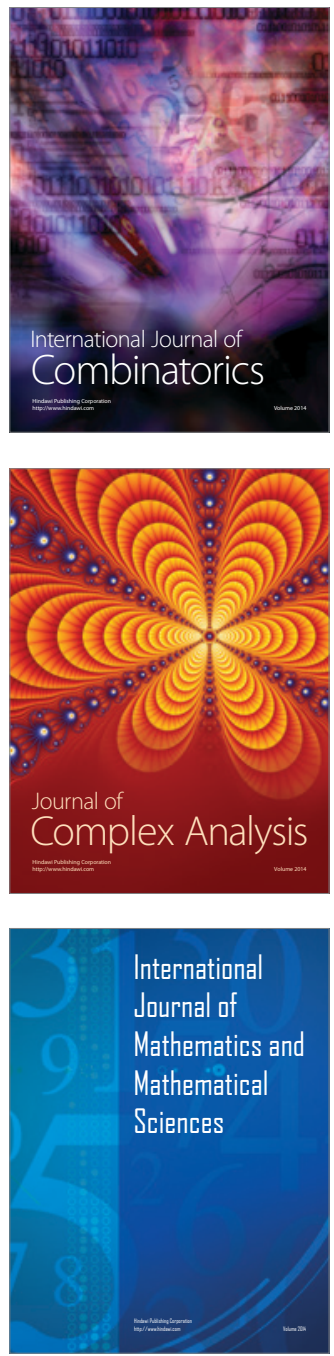
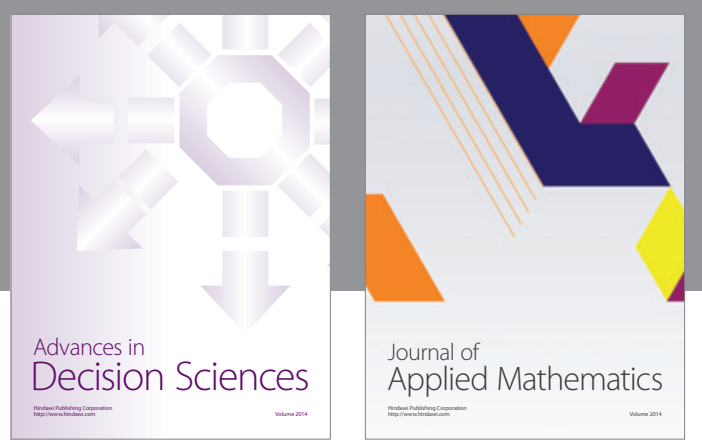

Algebra

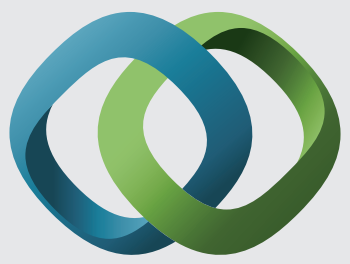

\section{Hindawi}

Submit your manuscripts at

http://www.hindawi.com


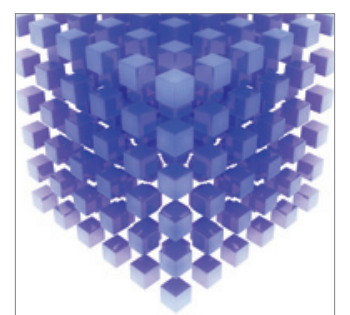

Mathematical Problems in Engineering


Journal of

Function Spaces



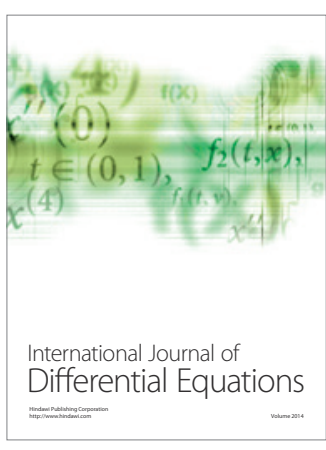
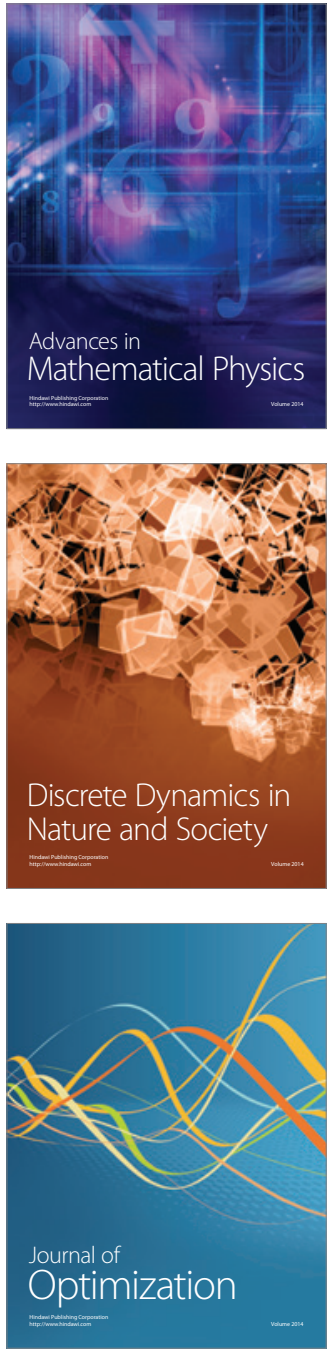